\title{
Multicenter experience with digital single-operator cholangioscopy in pregnant patients
}

\section{(ㄷ)(이우}

Authors

Olaya I. Brewer Gutierrez ${ }^{1}$, Gala Godoy Brewer ${ }^{1}$, Claudio Zulli², Sooraj Tejaswi ${ }^{3}$, Rishi Pawa ${ }^{4}$, Priya Jamidar ${ }^{5}$, Carlos Robles-Medranda ${ }^{6}$, Swati Pawa ${ }^{4}$, Jose V. Camilion7 ${ }^{7}$ Roberto Oleas ${ }^{6}$, Nasim Parsa ${ }^{8}$, Thomas Runge ${ }^{1}$, Diana Miaw $^{9}$, Yervant Ichkhanian ${ }^{1}$, Mouen A. Khashab ${ }^{1}$

Institutions

1 Division of Gastroenterology and Hepatology, Johns Hopkins Medical Institution, Baltimore, Maryland, United States

2 Division of Gastroenterology, University Hospital G. Fucito, Ruggi d'Aragona, Salermo, Italy

3 Division of Gastroenterology \& Hepatology, University of California Davis School of Medicine, Sacramento California, United States

4 Division of Gastroenterology, Wake Forest Baptist Medical Center, Winston Salem, NC

5 Division of Digestive Diseases, Yale University School of Medicine, New Haven, Connecticut, United States

6 Division of Gastroenterology and Endoscopy Division, Instituto Ecuatoriano de Enfermedades Digestivas, Guayaquil, Ecuador

7 Nova Southeastern College of Ostheopathic Medicine, Davie, Florida, United States

8 Division of Gastroenterology and Hepatology, University of Missouri, Columbia, MO

9 Division of Gynecology \& Obstetrics, Johns Hopkins Medical Institution, Baltimore, Maryland, United States

submitted 8.5 .2020

accepted after revision 19.10 .2020

Bibliography

Endoscopy International Open 2021; 09: E116-E121

DOI 10.1055/a-1320-0084

ISSN 2364-3722

(c) 2021. The Author(s).

This is an open access article published by Thieme under the terms of the Creative Commons Attribution-NonDerivative-NonCommercial License, permitting copying and reproduction so long as the original work is given appropriate credit. Contents may not be used for commecial purposes, or adapted, remixed, transformed or built upon. (https://creativecommons.org/licenses/by-nc-nd/4.0/)

Georg Thieme Verlag KG, Rüdigerstraße 14, 70469 Stuttgart, Germany
Corresponding author

Olaya I. Brewer Gutierrez, Johns Hopkins Medical Institution, 4949 Eastern Avenue, A Building, Suite 5E, Baltimore, MD 21224, USA

Fax: $+1-4105027010$

obrewer1@jhmi.edu

\section{ABSTRACT}

Background and study aims The use of fluoroscopy during pregnancy should be minimized given that a clear-cut safe radiation dose in pregnancy is unknown. The role of digital single-operator cholangioscopy (D-SOC) as an alternative to standard enodoscopic retrograde cholangiopancreatography (ERCP) in pregnant patients has not been comprehensively studied. This study assessed [1] Technical success defined as performance of ERCP with D-SOC without the use of fluoroscopy in pregnant patients; [2] safety of D-SOC in pregnancy; and [3] maternal and neonatal outcomes after D-SOC during/after pregnancy.

Patients and methods This was an international, multicenter, retrospective study at 6 tertiary centers. Pregnant patients who underwent D-SOC for the treatment of bile duct stones and/or strictures were included.

Results A total of 10 patients underwent D-SOC. Indications for ERCP were choledocholithiasis, strictures, previous stent removal, and choledocholithiasis/stent removal. Bile duct cannulation without fluoroscopy was achieved in 10 of 10 patients ( $100 \%)$. Moreover, $50 \%$ of patients $(5 / 10)$ completed a fluoroless ERCP with D-SOC. Mean fluoroscopy dose and fluoroscopy time were $3.4 \pm 7.2 \mathrm{mGy}$ and $0.5 \pm 0.8$ $\mathrm{min}$, respectively. One case of mild bleeding and one case of moderate post-ERCP pancreatitis occurred. The mean gestational age at delivery was $36.2 \pm 2.6$ weeks. Median birth weight was $2.5 \mathrm{~kg}$ [IQR: 2.2-2.8]. No birth defects were noted.

Conclusion ERCP guided by D-SOC appears to be a feasible and effective alternative to standard ERCP in pregnant patients. It enables avoidance of radiation in half of cases. 


\section{Introduction}

Gallstone disease commonly leads to non-gynecologic interventions during pregnancy and can be a major cause of hospitalizations in the first year postpartum [1,2]. The incidence of gallstone disease is markedly increased in pregnancy, given the differences in gallbladder motility as well as cholesterol saturation in bile. Increased gallstone disease can be associated with harmful maternal and neonatal outcomes, including preterm birth, readmission, and morbidity and mortality (maternal and neonatal) $[3,4]$. The management of symptomatic disease during pregnancy is often non-surgical to avoid fetal and maternal compromise. However, patients treated conservatively have a readmission rate from $38 \%$ to $70 \%$ [5].

Although endoscopic retrograde cholangiopancreatography (ERCP) is effective in treating common bile duct (CBD) stones during pregnancy [6-9], the major concern has been the use of fluoroscopy, which can expose the patient and fetus to significant radiation. A radiation exposure of 100 to 200 rads has been associated with major developmental anomalies [10-12]. During an ERCP procedure, the estimated radiation exposure dose to the fetus ranges from 0.1 to $3 \mathrm{mSv}(10 \mathrm{mSv}=1 \mathrm{rad})$ as a result of scattered radiation absorbed by the mother's body. While this dose may be lower than the one described to cause definite malformations, a safe radiation dose during pregnancy is still unknown and represents a risk, especially for potential DNA damage leading to genetic anomalies and cancer [8]. Therefore, non-fluoroscopic procedures that can allow the treatment of biliary disease are desirable $[13,14]$. ERCP with cholangioscopy, which has gained acceptance in the management of difficult bile duct stones and biliary strictures, can be potentially useful to avoid the use of radiation in pregnant patients who need ERCP. However, there is scarce literature describing the utility and outcomes of this technique in pregnancy, with most of the studies describing very small cohorts of patients and mostly utilizing non-digital SOC [15-17]. Herein, we present our multicenter experience with use of digital singleoperator cholangioscopy (D-SOC; Spyglass, Boston Scientific, Natick, Massachusetts, United States) for management of biliary disease in pregnant patients.

\section{Patients and methods}

This was a retrospective, international, multicenter study performed at six tertiary care centers from September 2015 to February 2020. Institutional Review Board (IRB) approval was obtained at all institutions. Medical records from female patients $>18$ years old who underwent ERCP with D-SOC (Spyglass, Boston Scientific, Natick, Massachusetts, United States) for biliary disease during pregnancy were queried.

The study included pregnant patients who required ERCP for biliary diseases. Non-pregnant patients were excluded from the study.

\section{Cholangioscopy-guided ERCP}

The fluoroless ERCP technique is as follows. Once the major papilla is identified, a sphincterotome preloaded with a
$0.025 / 0.035$-inch guidewire is used for a selective biliary cannulation. Once engaged, the wire is advanced, and if no resistance is encountered, it is followed by the sphincterotome. Aspiration of bile content confirms successful bile duct cannulation without the need for fluoroscopy. Once biliary cannulation is confirmed, the wire is advanced until resistance is met. Other authors have described the use of enddoscopic ultrasound prior to ERCP to determine the distance from the papilla to the bile duct bifurcation, so the wire can be advanced based on prior distance calculation. Then a biliary sphincterotomy is performed in a standard fashion, using Endocut I setting, when required by the presence of biliary stones or to facilitate stent placement followed by the passage of the digital cholangioscope into the bile duct. Careful cholangioscopy is then performed to assess the intrahepatic and extrahepatic bile ducts. If stones are seen, the cholangioscope is removed and the stone is retrieved wth standard techniques, such as an extraction balloon. If a stricture is encountered, then a miniforceps is advanced through the cholangioscope channel and direct biopsies are taken. If a stent is required, then the wire is advanced under cholangioscopic guidance into the intrahepatic ducts and the stent is deployed over the wire.

\section{Outcomes and definitions}

The primary outcome was to determine technical success, defined as the ability to perform cholangioscopy-guided ERCP without the use of fluoroscopy. Secondary outcomes included clinical success, defined as resolution of initial symptoms; safety, defined by the rate and severity of maternal adverse events (AEs) graded per the American Society for Gastrointestinal Endoscopy lexicon classification (mild, moderate, severe, fatal) [18]; and maternal and neonatal outcomes after D-SOC during and after pregnancy. Other outcomes included improvement in liver function tests (LFTs); quality of cholangioscopy visualization rated as poor, good or excellent by the performing endoscopist; fluoroscopy dose; fluoroscopy time; and total procedure time, defined by the time from scope-in to scope-out. Fluoroscopy time was defined as the cumulative time (in minutes) that fluoroscopy was used. Similarly, fluoroscopy dose was defined as the cumulative dose of radiation used during ERCP in milligrays (mGy) ( $1 \mathrm{mGy}=0.1$ rads).

\section{Statistical analysis}

Descriptive statistics was used for the analysis. Patient and disease characteristics were presented with percentages (\%), means \pm standard deviation (SD) and medians [IQR] according to the case. Statistical analysis was performed using SPSS Statistical Software ver. 22.0 (IBM, Armonk, New York, United States).

\section{Results}

A total of 10 patients underwent D-SOC while pregnant during the study period ( $\triangleright$ Table 1 ). The mean age was $26.8 \pm 4$ years, and the mean gestational age at the time of the procedure was $23.2 \pm 8.5$ weeks. Clinical presentation was as follows: abdominal pain $(n=6)$, cholangitis $(n=1)$ and asymptomatic $(n=3)$. 


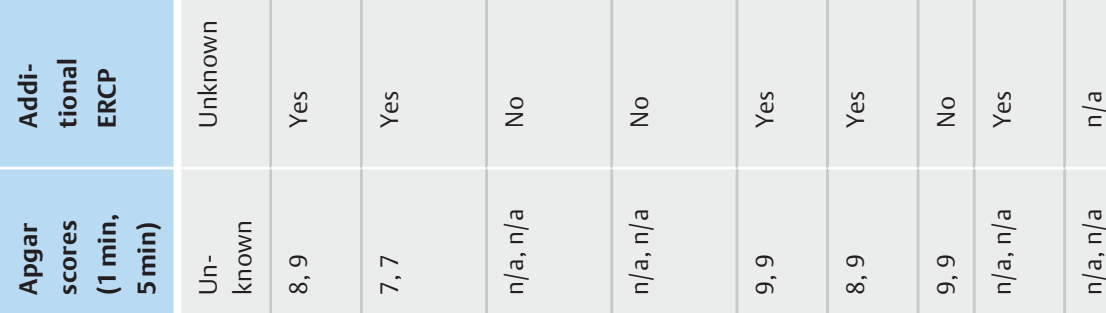

\begin{tabular}{|c|c|c|c|c|c|c|c|c|c|}
\hline$\frac{\vec{z}}{\bar{a}}$ & $\begin{array}{l}\frac{3}{3} \\
0 \\
\overline{5} \\
5\end{array}$ & $\stackrel{\check{\nu}}{\nu}$ & $\stackrel{\check{\nu}}{\check{\nu}}$ & 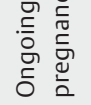 & 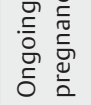 & $\stackrel{\check{\varpi}}{\succ}$ & $\stackrel{\check{\nu}}{\nu}$ & $\stackrel{\check{\nu}}{\nu}$ & 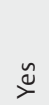 \\
\hline
\end{tabular}

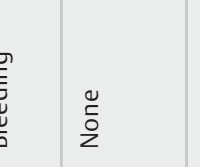

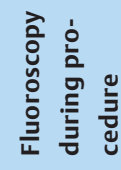

\begin{tabular}{|l|l|}
\hline & -1 \\
\hline
\end{tabular}

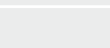


Most patients (70\%) had a prior cholecystectomy; in addition, more than half of the patients (60\%) underwent a prior ERCP, of whom two had a prior stent placed. Indications for previous ERCP were choledocholithiasis $(n=5)$ and stricture $(n=1)$. The patient with the stricture had a history of primary sclerosing cholangitis (PSC).

Indications for current ERCP were choledocholithiasis $(n=7)$, stent removal $(n=1)$, biliary stricture $(n=1)$, and choledocholithiasis/stent removal $(n=1)$. One patient was contacted to undergo an ERCP to remove a stent that was placed more than 1 year before the index ERCP and was lost to follow-up. Liver function tests were obtained from eight patients at the time of presentation, with a median aspartate transaminase, alanine aminotransferase, total bilirubin, and alkaline phosphatase of 57 IU/L [IQR: 39-73.5], 55IU/L [IQR: 40.5-83], 0.6 md/dL [IQR: $0.5-0.6]$, and $137 \mathrm{IU} / \mathrm{L}$ [IQR: 109-197.5], respectively.

Regarding details of the procedure, half of the patients received antibiotic prophylaxis during D-SOC. Successful bile duct cannulation without fluoroscopy was achieved in all patients ( $\mathbf{F i g . 1 )}$ ). Sphincterotomy was performed in five of 10 $(50 \%)$ cases. D-SOC was feasible in all patients. The quality of cholangioscopy visualization was rated as excellent by $40 \%$ of the performing physicians and as good by $60 \%$. Cholangioscopy findings were stones $(n=6)$, strictures $(n=1)$, sludge $(n=3)$, and a cholecystocholedocal fistula (Mirizzi syndrome) $(n=1)$. The location of the stricture was in the CBD, while the stone location was as follows: CBD $(n=4)$, cystic duct $(n=1)$, and in the right main intrahepatic duct $(n=1)$. The cholecystocholedochal fistula was located at the level of the mid-CBD. The mean stone size and median stone number were $5.2 \pm 2.7 \mathrm{~mm}$ and 2 [IQR: 1.5-4.5], respectively. One patient had a stone impacted in the right intrahepatic duct. Devices used for stone extraction were an extraction balloon in most of the cases $(n=4)$ and a retrieval basket $(n=2)$. A stent was placed in six patients $(60 \%)$. The patient with a history of PSC had a 70-mm-long stricture. Stricture brushing was performed, and pathology showed no malignancy. Successful stricture dilation to $6 \mathrm{~mm}$, under fluoroscopy, was completed. The Mirizzi syndrome was confirmed by visualization of the gallbladder mucosa during cholangioscopy. The stone was removed using an extraction balloon, and a stent was placed. Half the patients required the use of some fluoroscopy during the procedure.

Mean fluoroscopy dose and fluoroscopy time were $3.4 \pm$ $7.2 \mathrm{mGy}$ and $0.5 \pm 0.8 \mathrm{~min}$, respectively. The mean total procedure time was $40.3 \pm 24 \mathrm{~min}$. The median length of hospital stay was 2 days [IQR: 0-4]. Five patients required additional ERCPs with a median of one procedure [IQR: 0.3-1] for the following reasons: one patient underwent a post-pregnancy cholecystectomy complicated by a suspected biliary leak that required an additional ERCP, three patients underwent stent removal, and one patient experienced stent clogging and required stent replacement during pregnancy. Detailed patient characteristics and outcomes are described in > Table 1.
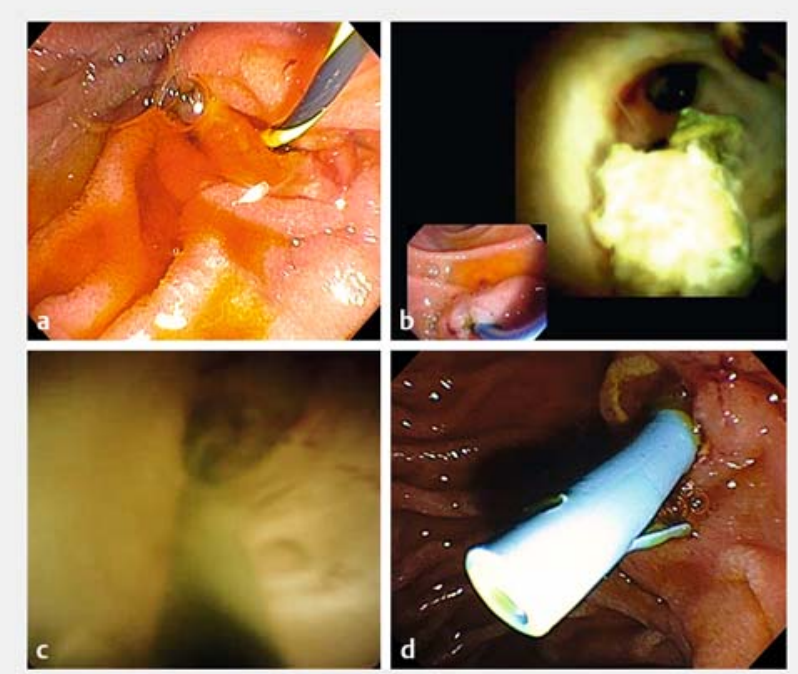

- Fig. 1 Fluoroless ERCP with D-SOC in a pregnant patient. a After selective cannulation and aspiration of bile to confirm biliary cannulation, the guidewire is gently advanced and then a standard sphincterotomy is performed. Bile is seen coming from the papilla. b The $\mathrm{D}-\mathrm{SOC}$ is advanced inside the CBD. Cholangioscopy is performed. The main right/left bifurcation is seen. Some sludge is noted. c A guidewire is advanced under cholangioscopy guidance into the right intrahepatic duct and exchanged with the cholangioscope. $\mathbf{d}$ Then a $10 \mathrm{~F} \times 7 \mathrm{~cm}$ plastic stent was deployed over the wire.

\section{Adverse events}

Two patients (20\%) presented with post-procedure AEs. One patient had post-ERCP bleeding managed conservatively and another patient developed post-ERCP pancreatitis that required extended hospitalization for 4 days with symptomatic management. The bleeding was classified as mild and pancreatitis as moderate per the ASGE Lexicon.

\section{Primary and secondary outcomes}

Technical success, defined as completion of the ERCP and DSOC without the use of fluoroscopy, was achieved in half the patients $(n=5,50 \%)$. Clinical success was reported in $100 \%$ of patients.

\section{Maternal outcomes}

The mean gestational age at delivery was $36.2 \pm 2.6$ weeks. Two patients (20\%) had preterm labor 3 and 8 weeks after undergoing ERCP with D-SOC at 33 and 34 weeks of gestation. Information regarding delivery method was obtained in five patients; three of five patients had a normal vaginal delivery while two of five had a cesarean section. There was no reported spontaneous abortion or intrauterine fetal demise. Two patients experienced post-delivery complications: one patient developed preeclampsia and another post-partum hemorrhage. Three patients were still pregnant at the conclusion of the study. One patient underwent ERCP with stent replacement due to stent clogging 1 week after the index ERCP and after 1 month, she developed right upper quadrant pain with distended gallblad- 
der with sludge and stones on transabdominal ultrasound. The patient underwent percutaneous cholecystostomy tube placement. Three days later, she presented with features of preeclampsia and underwent a cesarean section at 33 weeks of gestation. To date, two patients have undergone postpartum stent removal at a median of $19.5 \pm 23.3$ months post-delivery.

\section{Neonatal outcomes}

Neonatal outcomes were obtained in five newborns. The mean birth weight was $2.5 \mathrm{~kg}$ [IQR: 2.2-2.8]; two newborns had low birth weight (defined as $2.5 \mathrm{~kg} / 5.5 \mathrm{lb}$ or less by the World Health Organization (WHO)). The mean Apgar scores at 1 and 5 minutes were $8.2 \pm 0.8$ and $8.6 \pm 0.9$, respectively. One newborn required post-delivery admission to the neonatal intensive care unit (NICU) while the mother experienced postpartum hemorrhage. No neonate presented with birth defects. Information about developmental milestones was obtained from two neonates, with no documented delay.

\section{Discussion}

The use of ionizing radiation during ERCP is discouraged in pregnant patients due to the unknown safe cutoff of the radiation dose and potentially harmful effects on the fetus, especially during the first trimester. Exposure of less than $1 \mathrm{mGy}$ is recommended during the first trimester, and exposure of less than $5 \mathrm{mGy}$ is recommended during the entire gestation, but a clear safe dose of radiation during pregnancy is unknown [19]. Total radiation time and dosage are the two most important factors during fetal exposure that can be minimized with lead shielding. However, the principal source of radiation affecting the fetus is scattered radiation, mainly absorbed by the mother's body [13]. The use of techniques such as balloon dilation for biliary stone removal during pregnancy have shown to be effective; however, the high rate of post-ERCP pancreatitis makes unsafe to be routinely recommended $[20,21]$, probably due to the occurrence of a sphincter of Oddi spasm or post-endoscopic biliary balloon dilation peri-papillary edema [22]. DSOC represents an alternative to standard ERCP in pregnant patients, because it provides direct and good visualization of the extrahepatic biliary tree during ERCP, reducing the need for or the dose of ionizing radiation.

There are no published data on the use of the digital version of SOC and few published data on the use of the fiberoptic version in pregnant patients. Shelton et al performed a retrospective review of consecutive ERCPs done on five pregnant patients using SOC to confirm stone clearance. The authors reported clear visualization of the biliary tree without difficulty. They also reported no complications related to the use of cholangioscopy in those patients [16]. Sethi et al published a case report of a 24-year-old and 28-week pregnant patient who presented with right upper quadrant pain and elevated LFTs. An ultrasound showed dilation of the CBD to $10 \mathrm{~mm}$. The patient underwent EUS followed by ERCP; EUS demonstrated a 4-mm ductal dilation with multiple galltones in the mid-CBD. ERCP without fluoroscopy was performed and SOC was then used to confirm ductal clearance. An impacted stone at the junction of the cystic and bile duct was identified and removed using an extraction balloon. There were no reported complications [17].

In the current report, we demonstrated that the digital version of SOC is a potentially safe and effective alternative to standard ERCP. It can be used to diagnose and treat biliary stones and strictures, as well as to confirm the absence of residual stones, reducing radiation exposure in pregnant patients and the fetus. Bile duct cannulation without fluoroscopy was achieved in $100 \%$ of the patients with $50 \%$ having a completely fluoroless procedure. In those patients that required use of fluoroscopy, the dose was minimized below the recommended dose in three of five patients. The mean fluoroscopy dose and fluoroscopy time were $3.4 \pm 7.2 \mathrm{mGy}$ and $0.5 \pm 0.8 \mathrm{~min}$, respectively, which represents a minimal exposure.

Our study has several limitations. First and most importantly, it was retrospective with a small cohort. Second, some data were unavailable from a few centers. Finally, all cases were performed by experienced endoscopists at tertiary care centers, limiting the generalizability of the results. Nevertheless, to our knowledge, our multicenter experience represents the largest cohort published to date on the use of D-SOC in lieu of standard ERCP in pregnant patients. Larger cohorts are needed to define the safety and efficacy of this technique in this patient population.

\section{Conclusion}

In conclusion, ERCP with D-SOC might represent an efficient, safe, and minimally invasive technique for management of biliary disease, potentially reducing maternal and fetal exposure to ionizing radiation and its associated complications.

\section{Competing interests}

Dr. Jamidar is a speaker for Boston Scientific. Dr. Robles-Medranda is a key opinion leader and consultant for Pentax Medical, Boston Scientific, G-Tech medical supply, and MD Consulting group. Dr. Khashab is a consultant for Boston Scientific, Medtronic, and Olympus.

\section{References}

[1] Valdivieso V, Covarrubias C, Siegel F et al. Pregnancy and cholelithiasis: pathogenesis and natural course of gallstones diagnosed in early puerperium. Hepatology 1993; 17: 1-4

[2] IIlhan M, ilhan G, Gök AFK et al. The course and outcomes of complicated gallstone disease in pregnancy: Experience of a tertiary center. Turk J Obstet Gynecol 2016; 13: 178-182

[3] Ibiebele I, Schnitzler M, Nippita T et al. Outcomes of gallstone disease during pregnancy: a population-based data linkage study. Paediatr Perinat Epidemiol 2017; 31: 522-530

[4] Bowie JM, Calvo RY, Bansal V et al. Association of complicated gallstone disease in pregnancy and adverse birth outcomes. Am J Surg 2020; 220: 745-750

[5] Date RS, Kaushal M, Ramesh A. A review of the management of gallstone disease and its complications in pregnancy. Am J Surg 2008; 196: 599-608 
[6] Tang SJ, Mayo M], Rodriguez-Frias E et al. Safety and utility of ERCP during pregnancy. Gastrointest Endosc 2009; 69: 453-461

[7] Zhou Y, Zhang X, Guo Y et al. ERCP in acute cholangitis during third trimester of pregnancy. Hepatogastroenterology 2013; 60: 981-984

[8] Tham TC, Vandervoort J, Wong RC et al. Safety of ERCP during pregnancy. Am J Gastroenterol 2003; 98: 308-311

[9] Arce-Liévano E, Del Río-Suárez I, Valenzuela-Salazar C et al. Endoscopic retrograde cholangiopancreatography results for the treatment of symptomatic choledocholithiasis in pregnant patients: A recent experience at a secondary care hospital in Mexico City. Rev Gastroenterol Mex 2020: doi:10.1016/j.rgmx.2019.12.001

[10] Baron TH, Schueler BA. Pregnancy and radiation exposure during therapeutic ERCP: time to put the baby to bed? Gastrointest Endosc 2009; 69: 832-834

[11] McCollough CH, Schueler BA, Atwell TD et al. Radiation exposure and pregnancy: when should we be concerned? Radiographics 2007; 27: 909-917

[12] Sternberg J. Radiation and pregnancy. Can Med Assoc J 1973; 109: $51-57$

[13] Samara ET, Stratakis J, Melono Enele JM et al. Therapeutic ERCP and pregnancy: is the radiation risk for the conceptus trivial? Gastrointest Endosc 2009; 69: 824-831

[14] Savas N. Gastrointestinal endoscopy in pregnancy. World J Gastroenterol 2014; 20: 15241-15252
[15] Girotra M, Jani N. Role of endoscopic ultrasound/SpyScope in diagnosis and treatment of choledocholithiasis in pregnancy. World J Gastroenterol 2010; 16: 3601-3602

[16] Shelton J, Linder JD, Rivera-Alsina ME et al. Commitment, confirmation, and clearance: new techniques for nonradiation ERCP during pregnancy (with videos). Gastrointest Endosc 2008; 67: 364-368

[17] Sethi S, Thosani N, Banerjee S. Radiation-free ERCP in pregnancy: a "sound" approach to leaving no stone unturned. Dig Dis Sci 2015; 60: 2604-2607

[18] Cotton PB, Eisen GM, Aabakken L et al. A lexicon for endoscopic adverse events: report of an ASGE workshop. Gastrointest Endosc 2010; 71: $446-454$

[19] Chan CH, Enns RA. ERCP in the management of choledocholithiasis in pregnancy. Curr Gastroenterol Rep 2012; 14: 504-510

[20] Baron TH, Harewood GC. Endoscopic balloon dilation of the biliary sphincter compared to endoscopic biliary sphincterotomy for removal of common bile duct stones during ERCP: a metaanalysis of randomized, controlled trials. Am J Gastroenterol 2004; 99: 1455-1460

[21] Weinberg BM, Shindy W, Lo S. Endoscopic balloon sphincter dilation (sphincteroplasty) versus sphincterotomy for common bile duct stones. Cochrane Database Syst Rev 2006; 4: CD004890

[22] Arnold JC, Benz C, Martin WR et al. Endoscopic papillary balloon dilation vs. sphincterotomy for removal of common bile duct stones: a prospective randomized pilot study. Endoscopy 2001; 33: 563-567 